\title{
PSYCHIATRIC PATIENTS ALSO TRAVEL AROUND THE WORLD: WHAT WE SHOULD KNOW TO HELP THEM TRAVELING SAFE
}

\section{G. Baquero Mahecha 1 .}

${ }^{1}$ EuroCenter - Madrid S.A., Medical, Madrid, Spain.

Objectives: The author wants to bring awareness about the need to support psych-patients when travelling.

Background: Psychiatric patients experience troublesome situations due to lack of preparation of both doctors and themselves, when travelling.

As psychiatrists we should help our patients to travel safely as a normal part of their daily life, providing a plan for continuous treatment, prevention and how to react in case of unexpected difficult scenarios.

Materials and Methods: Through the presentation of cases, the author will report real situations that have faced patients while traveling due to the lack of preparation, misunderstandings and insufficient communication between doctors and then suggest a strategy to avoid recurrence of such situations.

Results and Conclusions: Lack of communication between foreign treating doctor, home psychiatrist, travel insurance, and the patient himself are detrimental for a safe travel experience. Guidelines of conduct and treatment should be written and diffused worldwide to specialist and non-specialist physicians, in order to reduce the incidence of emergency scenarios and include travelling into the list of common activities of a psych patient.

Keywords: Travel, Guideline, Treatment, Repatriation.

People travels to relax, to disconnect, to get to know different places, but this also implies a level of stress. That stress can increase if unexpected situations such robbery, accidents or other situations happen and either trigger or worsen an acute episode.

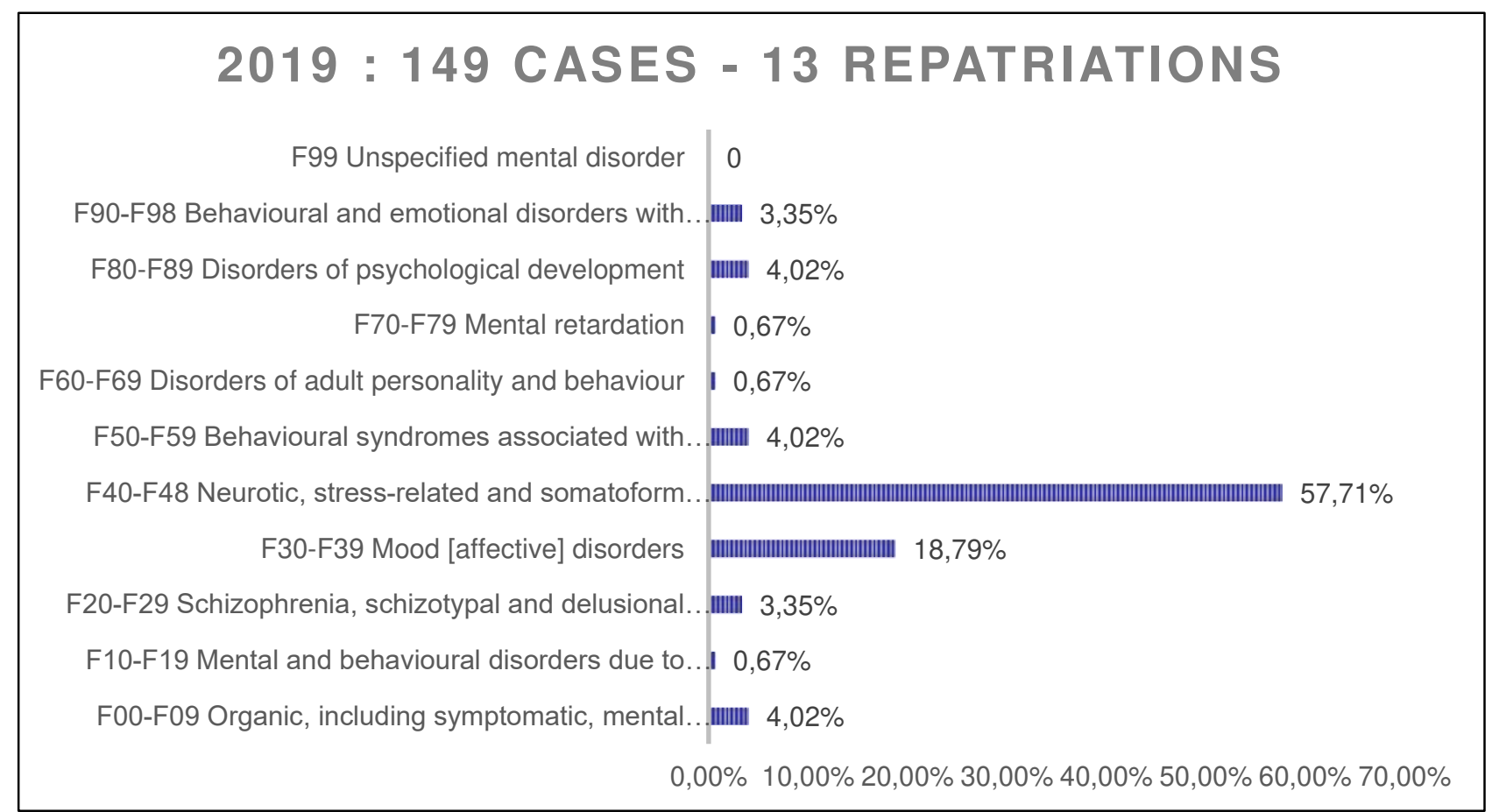

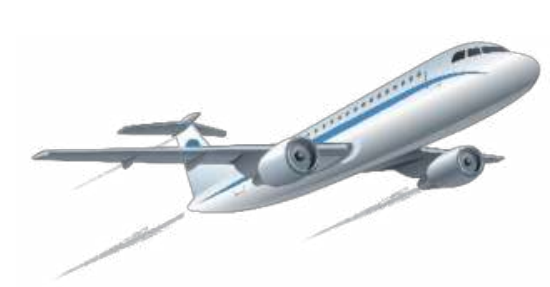

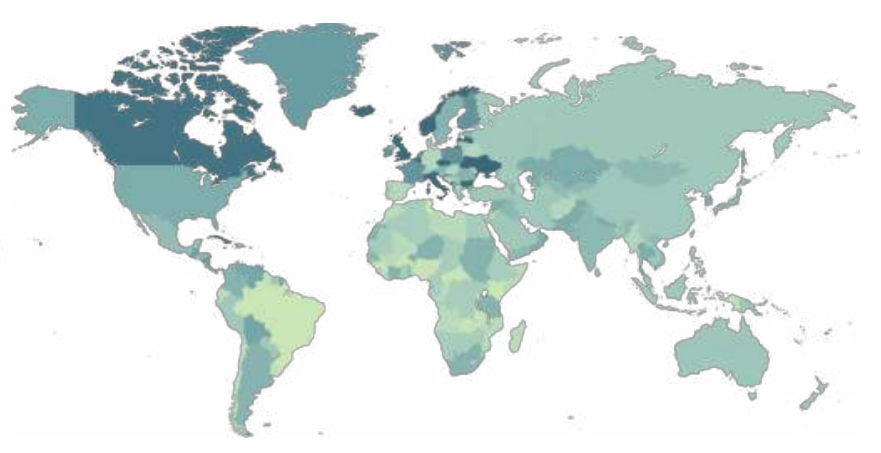

For example: being robbed triggered a psychotic outbreak of a schizophrenic patient. A patient with OCD suffered a severe relapse of the obsessions and compulsions, he stopped the treatment because he feared he was contaminated during a routine customs check upon arrival to destination. A teenager patient that once was on a scholar travel, opened to her teacher about structured suicide ideation related to depressive disorder later discovered to be on the context of sexual abuse. After a motor-vehicle accident a patient developed an organic personality disorder. Cases like these were reported to the travel insurance : A total of 149 psychiatric cases out of 13955 total claims in 2019, 173 psychiatric cases out of 19046 total claims in 2018, 177 psychiatric cases out of 18263 total claims in 2017 and 60 psychiatric cases out of 21896 total claims in 2016.

Occasionally, it was necessary to repatriate these patients escorted by doctors and in some cases also with a nurse due to the severity of the symptoms. $(1,2)$ As most of the travels are made by plane, the upon-mentioned lack of communication, exposes the medical team, the patient and the other passengers to complex scenarios that could also include emergency landing.

In conclusion, in order to reduce, control or even prevent repatriations, worldwide guidelines about travelling-psych patients should be published.

Most of the bibliographic sources is older than 20 years. Therefore, it is paramount to update a new consensus for $21^{\text {st }}$ century's travelers.

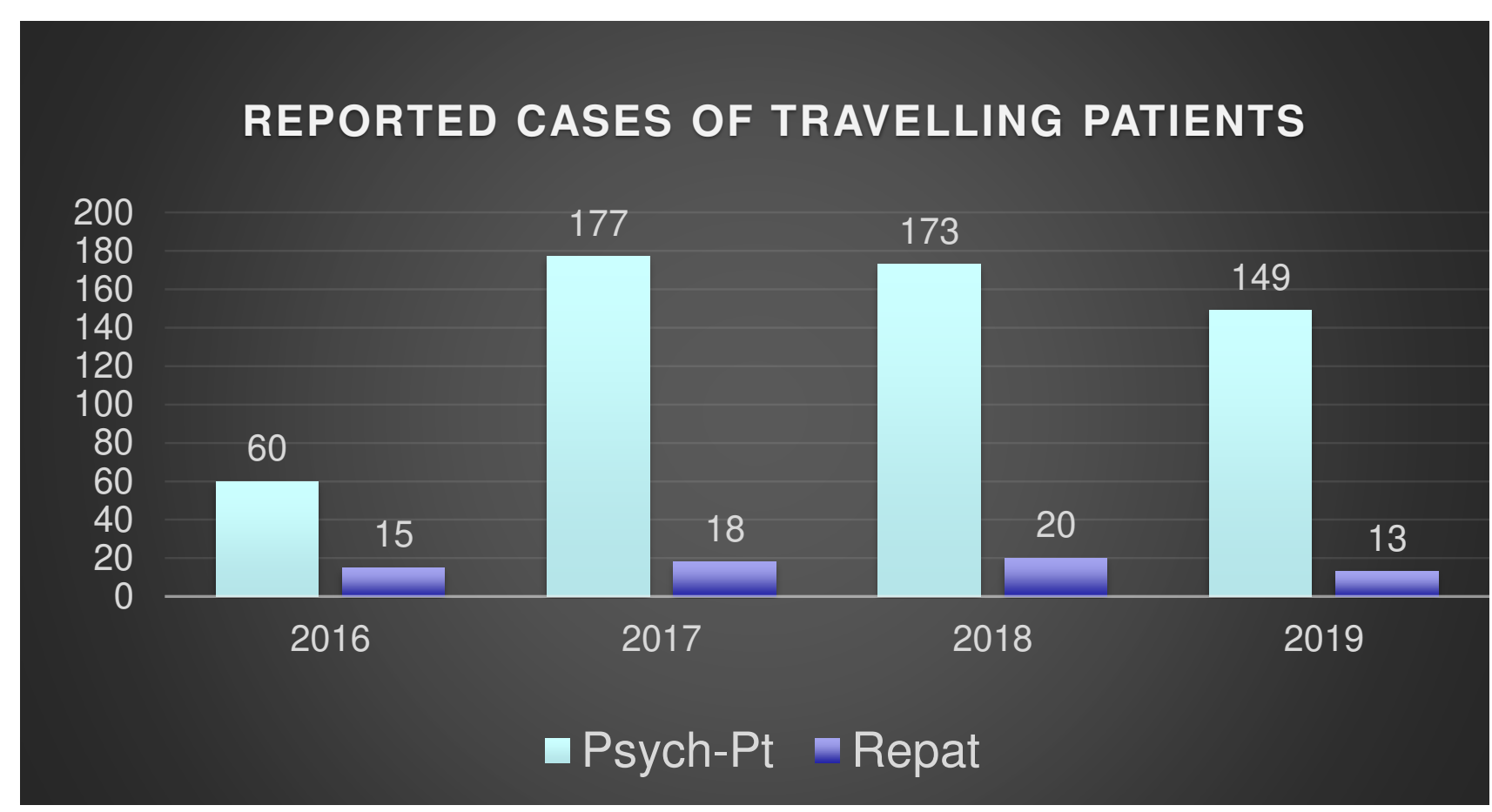

